\title{
THERMAL COLOR-SUPERCONDUCTING FLUCTUATIONS IN DENSE QUARK MATTER
}

\author{
D.N. Voskresensky \\ Gesellschaft fur Schwerionenforschung mbH, Planckstr. 1, 64291 Darmstadt, Germany; \\ Moscow Institute for Physics and Engineering, Kashirskoe sh. 31, Moscow 115409, Russia \\ D.Voskresensky@gsi.de
}

\begin{abstract}
Thermal fluctuations of the color superconducting order parameter in dense quark matter are investigated in terms of the phenomenological Ginzburg - Landau approach. Our estimates show that fluctuations of the di-quark gap may strongly affect some of thermodynamic quantities even far below and above the critical temperature. If the critical temperature $T_{c}$ of the di-quark phase transition were rather high $(\gtrsim(50 \div 70) \mathrm{MeV})$ one could expect a manifestation of fluctuations of the di-quark gap in the course of heavy ion collisions (above $T_{c}$ ). For $T_{c} \sim 50 \mathrm{MeV}$ color superconducting fluctuations may also affect an initial stage of the hybrid star evolution.
\end{abstract}

Keywords: color superconductivity, order parameter, temperature, fluctuations

\section{Introduction}

The quark-quark interaction in the color antitriplet channel is attractive driving the pairing, cf. [1]. The problem has been re-investigated in a series of papers following Refs. [2, 3], see review [4] and Refs therein. The attraction comes from the one-gluon exchange, or from a nonperturbative 4-point interaction motivated by instantons [5], or nonperturbative gluon propagators [6]. The zero-temperature pairing gap $\Delta$ was predicted to be $\sim(20 \div 200) \mathrm{MeV}$ for the quark chemical potentials $\mu_{q} \sim(300 \div 500) \mathrm{MeV}$. In the standard BCS theory, cf. [4], the critical temperature is estimated as $T_{c} \simeq 0.57 \Delta$.

One expects the di-quark condensate to dominate the physics at densities beyond the deconfinement/chiral restoration transition and below the critical temperature. Various phases are possible. E.g., the so called 2-color superconductivity (2SC) phase allows for unpaired quarks of one color. There may also exist a color-flavor locked (CFL) phase [7] for not too large value of the strange quark mass $m_{s}$, for $2 \Delta>m_{s}^{2} / \mu_{q}$, cf. [8], where the color superconductivity (CSC) is complete in the sense that the di-quark condensation produces a gap 
for quarks of all three colors and flavors. The values of the gap are of the same order of magnitude for $2 \mathrm{SC}$ and CFL phases, whereas relations between critical temperature and the gap might be different, $T_{c} \simeq 0.57 \Delta$ for $2 \mathrm{SC}$ and $T_{c} \simeq 0.7 \Delta$ for CFL phase [9]. There are also another possibilities, e.g., of the pairing in spin-one channel, for which the pairing gap proves to be small $\Delta \lesssim 1 \mathrm{MeV}$, see [9].

The high-density phases of QCD at low temperatures may exist in interiors of most massive neutron stars (so called hybrid stars containing the quark core and the hadron shell) affecting the cooling, rotation and magnetic field, cf. [10-12]. It is also possible to ask whether CSC is relevant for the terrestrial experiments? To produce a color superconducting matter in the laboratory one would need to cook a dense and not too hot baryon enriched matter. The nuclear matter prepared in heavy ion collisions at SIS, AGS, SPS or RHIC in all the cases has presumably not sufficiently high baryon density and, on the other hand, the matter prepared at SPS or RHIC is feasibly too hot in order to expect a manifestation of the CSC. The most relevant is probably the GSI "Compressed baryon matter" future heavy ion collision facility which may cook enough dense and not too hot state. Baryon densities up to ten normal nuclear matter density $\left(\lesssim 10 \rho_{0}\right)$ at temperatures $T \lesssim 170 \mathrm{MeV}$ are expected to be reached at an initial collision stage. It is supposed that the system is in the quark-gluon plasma state at such conditions. Then the system expands and cools down. In this process the temperature decreases up to $T \sim 140 \mathrm{MeV}$ at still rather high baryon density at an intermediate collision stage. Although the temperatures at relevant densities are most likely larger than the critical temperature $T_{c}$ of CSC, one may rise the question on a possible manifestation of the precursor phenomena of the CSC phase transition, if $T_{c}$ is rather high $\left(T_{c} \gtrsim(50 \div 70) \mathrm{MeV}\right)$. Recently, ref. [13] considered such a possibility within the Nambu-Jona-Lasinio model and demonstrated that the fluctuating pair field results in a prominent peak of the spectral function, which survives in the temperature interval $\left|T-T_{c}\right| \lesssim(0.1 \div 0.2) T_{c}$. Besides, it is interesting to investigate the role of the order parameter fluctuations for $T<T_{c}$, if $T_{c}$ is $\sim 50 \mathrm{MeV}$, that still may affect the neutrino radiation of the most massive hot neutron stars (if they indeed have quark cores) and the heat transport at an initial stage of their evolution.

This paper is an extended version of the work [14]. We will study precursor phenomena of the CSC in the framework of the phenomenological Ginzburg Landau approach. To be specific, we will consider condensates with total angular momentum $J=0$ that are antisymmetric in color and flavor. Such pairing states can occur in the weak coupling limit because one-gluon exchange is attractive in the color anti-triplet channel. Although for reasonable values of densities and temperatures conditions of the applicability of the weak coupling limit are hardly fulfilled, in order to get a feeling on possible relevance of fluc- 
tuation effects we will still use this limit. At the same time we will by hand vary the relation $\Delta\left(\mu_{q}\right)$, since the corresponding exponential dependence is most sensitive to corrections of the running QCD coupling constant compared to its perturbative value. We will restrict our discussion by the consideration of phases, which feasibly have large gaps, like 2SC and CFL, as the most interesting case for applications.

\section{Physics of pairing fluctuations}

Like there always exists a vapor under the water, there are excitations on the ground of any condensate. They appear due to quantum and thermal fluctuations. In classical systems and also at not too small temperatures in quantum systems, quantum fluctuations are suppressed compared to thermal fluctuations. Excitations are produced and dissolved with the time passage, although the mean number of them is fixed at given temperature. Pairing fluctuations are associated with formation and breaking of excitations of a particular type, Cooper pairs out of the condensate. Fluctuation theory of phase transitions is a well developed field. In particular, ten thousands of papers in condensed matter physics are devoted to the study of pairing fluctuations. At this instant we refer to an excellent review of Larkin and Varlamov [15].

In some phenomena pairing fluctuations behave similarly to quasiparticles. However there are also differences:

- Typical "binding energy" $E_{\text {bind }}$ of pairing fluctuation is of the same order as inverse life-time $\tau_{\text {l.t. }}^{-1} \propto 1 /\left|T-T_{c}\right|$, whereas for quasiparticles $E \gg \tau_{\text {l.t. }}^{-1}$.

- Typical size $l \propto 1 / \sqrt{\left|T-T_{c}\right|}$ is large for $T$ near $T_{c}$. Quasiparticles have a small typical size.

- Fluctuations near $T_{c}$ behave as classical fields in sense of RayleighJeans: 3-momentum distribution is $n(p) \sim T / E(p)$ in the vicinity of $T_{c}$.

We will demonstrate below that the Ginzburg number $\left(G i=\Delta T / T_{c}\right)$, which determines the broadness of the energy region near the critical temperature, where fluctuations essentially contribute, is $G i \sim A\left(T_{c} / \mu_{q}\right)^{4}$ with $A \sim 500$ in our case. To compare, for clean metals $A \sim 100, \mu_{q} \rightarrow \mu_{e}$, the latter is the electron chemical potential. Thus $G i \sim 1$, if $T_{c}$ is rather high,

$T_{c} \sim\left(\frac{1}{3} \div \frac{1}{5}\right) \mu_{q}$, and we expect a broad region of temperatures, where fluctuation effects might be important. 


\section{Thermodynamical potential and its mean field solution}

The Fourier component of the density of the thermodynamic potential (thermodynamic potential per unit volume $V$ ) in the superconducting quark matter with the di-quark pairing can be written in the following form $[12,16]$, cf. also $[1,11]$,

$$
\begin{aligned}
\widetilde{\Omega} & =\widetilde{\Omega}_{n}+\sum_{\alpha, i}\left(-c_{0}\left|\partial_{\tau} d_{\alpha}^{i}\right|^{2}+c\left|\nabla d_{\alpha}^{i}\right|^{2}\right)+a D+\frac{b}{2} D^{2}, \\
D & =\sum_{\alpha, i}\left|d_{\alpha}^{i}\right|^{2}, \quad \gamma=\frac{1}{D^{2}} \sum_{\alpha, \beta, i}\left|d_{\alpha}^{i, *} \cdot d_{\beta}^{i}\right|^{2}, \quad b=b_{1}+\gamma b_{2} .
\end{aligned}
$$

The Greek indices $\alpha, \beta=\{R, B, G\}$ count colors, the Latin indices $i=$ $\{u, d, s\}$ count flavors. The expansion is presented up to the fourth order in the di-quark field operators (related to the gap) assuming the second order phase transition, although at zero temperature the transition might be of the first order, cf. [17]. $\widetilde{\Omega}_{n}$ is the density of the thermodynamic potential of the normal state. The order parameter squared is $D=\left|\vec{d}_{\mathrm{IS}}\right|^{2}=\left|\vec{d}_{R}\right|^{2}+\left|\vec{d}_{G}\right|^{2}+\left|\vec{d}_{B}\right|^{2}$, $\vec{d}_{R}\left\|\vec{d}_{G}\right\| \vec{d}_{B}$ for the isoscalar phase (IS), and $D=3\left|\vec{d}_{\mathrm{CFL}}\right|^{2},\left|\vec{d}_{R}\right|^{2}=\left|\vec{d}_{G}\right|^{2}=$ $\left|\vec{d}_{B}\right|^{2}=\left|\vec{d}_{\mathrm{CFL}}\right|^{2}, \quad \vec{d}_{R}^{*} \cdot \vec{d}_{G}=\vec{d}_{G}^{*} \cdot \vec{d}_{B}=\vec{d}_{B}^{*} \cdot \vec{d}_{R}=0$ for the CFL phase, $\vec{d}_{\alpha}=\left\{d_{\alpha}^{u}, d_{\alpha}^{d}, d_{\alpha}^{s}\right\}$. The so called 2SC phase is the IS phase with unpaired quarks of one color (to say $R$ ). The interaction of the di-quark field with fluctuation gluon fields is usually introduced in the standard way through the corresponding gauge-shifted full co-variant derivatives. Recent paper [18] demonstrated that the gluon fluctuations contribute essentially to thermodynamic quantities only at temperatures in a narrow vicinity of the critical temperature, $\Delta T / T_{c} \lesssim 0.1$ for relevant values of parameters. In this rather small temperature interval, $\Delta T$, gluon field fluctuations change the nature of the phase transition (from the second to the first order). As we will show below, the value $\Delta T$ is much less than the temperature region, where fluctuations of the di-quark order parameter might be important. Thereby in the latter discussion we suppress the gluon fields as well as the discussion of any peculiarities of this narrow temperature region near $T_{c}$.

Near the critical point coefficients of (1) can be expanded in $t=(T-$ $\left.T_{c}\right) / T_{c}$. In the weak coupling limit they render:

$$
\begin{aligned}
& a=a_{0} \ln \left(T / T_{c}\right) \simeq a_{0} t, \quad a_{0}=\frac{2 \mu_{q}^{2}}{\pi^{2}}, \\
& b_{1}=b_{2}=b /(1+\gamma)=\frac{7 \zeta(3) \mu_{q}^{2}}{8 \pi^{4} T_{c}^{2}}, \quad c=\frac{b}{3(1+\gamma)}, \quad c_{0}=3 c .
\end{aligned}
$$

For the classical (mean) fields $\gamma=1$ in the IS phase and $\gamma=1 / 3$ in the CFL phase, cf. [12], $\mu_{q}=\mu_{B} / 3, \mu_{B}$ is the baryon chemical potential (the contri- 
bution of the strange quark mass is neglected), $\zeta(3)=1.202 \ldots$ The critical temperature $T_{c}$ is the same for the IS and the CFL phases for $m_{s} \rightarrow 0$. For $m_{s} \neq 0, T_{c}$ would depend on $m_{s}$ that would result in a smaller $T_{c}$ for the CFL phase than for the IS phase.

The value of the order parameter follows by solving the equation of motion for the field operators, $\delta \widetilde{\Omega} / \delta d_{\alpha}^{i}=0$ :

$$
-c_{0} \partial_{\tau}^{2} d_{\alpha}^{i}+c \Delta d_{\alpha}^{i}-a d_{\alpha}^{i}-b D d_{\alpha}^{i}=0
$$

The stationary, spatially-homogeneous mean field solution of (3) (without taking into account of fluctuations) is

$$
D_{\mathrm{MF}}=-a \Theta(-t) / b, \quad \delta \widetilde{\Omega}_{\mathrm{MF}}=-\frac{a^{2}}{2 b} \Theta(-t),
$$

where the step function $\Theta(-t)=1$ for $t<0$ and $\Theta(-t)=0$ for $t>0$.

To be specific, discussing $T<T_{c}$ we further consider the CFL case. For the finite system of a large spherical size $R \gg \xi$, with $\xi$ having the meaning of the coherence length, we obtain

$$
d_{\alpha, \mathrm{MF}}^{i}= \pm \frac{\delta_{\alpha}^{i}}{\left(N_{c} N_{f}\right)^{1 / 4}} \sqrt{D_{\mathrm{MF}}} \Theta(-t) \text { th }\left[\frac{(R-r)}{\sqrt{2} \xi}\right], \quad \xi=\sqrt{\frac{c}{|a|}},
$$

where $N_{c}=3$ is the number of colors, $N_{f}=3$ is the number of flavors and to be specific we assumed the simplest structure $d_{\alpha, \mathrm{MF}}^{i} \propto \delta_{\alpha}^{i}$.

\section{Fluctuations of gap in self-consistent Hartree approximation}

Now we will consider fluctuations. Below $T_{c}$ we present $d_{\alpha}^{i}$, as $d_{\alpha}^{i}=d_{\alpha, c}^{i}+$ $d_{\alpha}^{i, \prime}$, and above $T_{c}$, as $d_{\alpha}^{i}=d_{\alpha}^{i,{ }^{\prime}}$, where index "c" labels the classical solution.

Since $\delta \widetilde{F}(V, T)=\delta \widetilde{\Omega}(\mu, T)$, cf. [19], the density of the free energy of the CFL phase expanded in $\left(d_{\alpha}^{i, \prime}\right)^{2}$ terms renders

$$
\begin{aligned}
\delta \widetilde{F} & =\delta \widetilde{F}_{c}+\delta \widetilde{F}^{\prime}=\sum_{\alpha, i}\left(-c_{0}\left|\partial_{\tau} d_{\alpha, c}^{i}\right|^{2}+c\left|\nabla d_{\alpha, c}^{i}\right|^{2}\right)+a D_{c} \\
& +\frac{b_{1}+b_{2} / 3}{2} D_{c}^{2}+\frac{5}{3}\left(b_{1}+b_{2} / 3\right) D_{c} N_{c} N_{f} \sum_{k}\left|\phi_{k}^{\prime}\right|^{2} \\
& +N_{c} N_{f} \sum_{k}\left(-c_{0} \omega^{2}+c \vec{k}^{2}+a\right)\left|\phi_{k}^{\prime}\right|^{2} \\
& +\nu_{H} \frac{b_{1}+b_{2}}{2}\left[N_{c} N_{f} \sum_{k}\left|\phi_{k}^{\prime}\right|^{2}\right]^{2} .
\end{aligned}
$$

The last term is introduced within the self-consistent Hartree approximation (within the $\Phi$ functional up to one vertex), $\nu_{H}=10 / 9$ accounts different 
coefficients in $\Phi$ functional for the self-interaction terms $\left(d^{4}\right.$ - for the given field $d$ and $\left(d_{\alpha}^{i}\right)^{2}\left(d_{\beta}^{j}\right)^{2}$ terms), cf [20]. We presented $d_{\alpha}^{i, \prime}=\sum_{k} d_{\alpha, k}^{i, \prime} e^{-i k^{\mu} x_{\mu}}$, $k^{\mu}=(\omega, \vec{k}), x_{\mu}=(\tau,-\vec{r})$, and introduced the notation

$$
\sum_{i, \alpha, k}\left|d_{\alpha, k}^{i, \prime}\right|^{2}=\sum_{k}\left|\phi_{k}^{\prime}\right|^{2}=i \int \frac{d^{4} k}{(2 \pi)^{4}} \frac{1}{c_{0} \omega^{2}-c \vec{k}^{2}-m^{2}} .
$$

In Matsubara technique the temperature dependence is introduced by the replacements: $\omega \rightarrow \omega_{n}=2 \pi i n T,-i \int \frac{d^{4} k}{(2 \pi)^{4}} \rightarrow T \sum_{n=-\infty}^{n=\infty} \int \frac{d^{3} k}{(2 \pi)^{3}}$. We restricted ourselves by the self-consistent Hartree approximation. Effects of the damping of fluctuations, being produced by the two- and more vertex diagrams of $\Phi$, are beyond the scope of this simple approximation. As we argue below, the main effects we discuss in this paper are not essentially affected by the width terms.

Variation of eq. (5) over $\phi_{k}^{\prime}$ yields the spectrum of fluctuations

$$
c_{0} \omega^{2}-c \vec{k}^{2}-m^{2}=0,
$$

with the squared mass parameter

$$
m^{2}=\eta|a|=\frac{5}{3}\left(b_{1}+b_{2} / 3\right) D_{c}+a+\nu_{H}\left(b_{1}+b_{2}\right) N_{c} N_{f} \sum_{k}\left|\phi_{k}^{\prime}\right|^{2} .
$$

Notice that the real physical meaning of the effective mass has the quantity $m / \sqrt{c_{0}}$ rather than $m$, as follows from (8).

Solution of the equation for the fluctuating field presented in the coordinate space is characterized by the length scale $l=\xi / \sqrt{\eta}$ and by the time scale $\widetilde{\tau}=$ $\xi c_{0}^{1 / 2}(c \eta)^{-1 / 2}$. Above the critical point $D_{c}=0, m^{2}=a+O\left(\sum_{k}\left|\phi_{k}^{\prime}\right|^{2}\right)>0$, and neglecting $\left|\phi_{k}^{\prime}\right|^{2}$ terms one gets the parameter $\eta \simeq 1$.

Variation of (5) over $d_{\alpha, c}^{i, *}$ yields the equation of motion for the classical field

$$
\begin{aligned}
& -c_{0} \partial_{\tau}^{2} d_{\alpha, c}^{i}+c \Delta d_{\alpha, c}^{i}-\left(a+\frac{5}{3}\left(b_{1}+b_{2} / 3\right) N_{c} N_{f} \sum_{k}\left|\phi_{k}^{\prime}\right|^{2}\right) d_{\alpha, c}^{i} \\
& -\left(b_{1}+b_{2} / 3\right) D_{c} d_{\alpha, c}^{i}=0 .
\end{aligned}
$$

Only dropping the term responsible for fluctuations we find $D_{c}=D_{\mathrm{MF}}$, $d_{\alpha, c}^{i}=d_{\alpha, \mathrm{MF}}^{i}, m^{2}=m_{\mathrm{MF}}^{2}=\eta_{\mathrm{MF}}|a|$ for $T<T_{c}$, cf. (4), (9), and we obtain $\eta_{\mathrm{MF}}=2 / 3$. In reality fluctuations affect the classical solution and renormalize the critical temperature of the phase transition $T_{c}$ yielding $T_{c}^{\text {ren }}<T_{c}$. More generally

$$
m^{2}=m_{\mathrm{MF}}^{2}+\delta m^{2}, \quad \delta m^{2}=-\frac{5}{3}\left(b_{1}-b_{2} / 9\right) N_{c} N_{f} \sum_{k}\left|\phi_{k}^{\prime}\right|^{2},
$$


for $T<T_{c}^{\mathrm{ren}}$. From (9), (11) one finds $D_{c}\left(m^{2}=0\right)=0$, as the consequence of the self-consistency of our approximation scheme. As we have mentioned, we neglected fluctuations of the gluon fields, which change the nature of the phase transition but yield only a small jump of the order parameter.

\section{Contribution of fluctuations of gap to specific heat below $T_{c}$}

In order to demonstrate how fluctuations of the order parameter may affect thermodynamic quantities let us calculate the contribution of fluctuations to the specific heat density $\widetilde{C}_{V}=-T\left(\frac{\partial^{2} \delta \widetilde{F}}{(\partial T)^{2}}\right)_{V}$, cf. [19]. The mean field contribution is

$$
\widetilde{C}_{V}^{\mathrm{MF}}=T \frac{a_{0}^{2}}{b T_{c}^{2}} \Theta(-t)
$$

as it follows from (1), (2) and (4). Here and below we use an approximate expression $a \simeq a_{0} t$ valid at $T$ near $T_{c}$, see (2). The contribution to the specific heat from the normal quark excitations is suppressed as $\propto T^{-3 / 2} e^{-\Delta(0) / T}$ for $T \ll T_{c}$, cf. [21]. We may reproduce correct low temperature behavior multiplying (12) by a form-factor $f \simeq 2 e^{-\Delta(0) / T}\left(T_{c} / T\right)^{5 / 2}\left(1-\frac{2 T}{3 T_{c}}\right)^{-1}$.

The fluctuation contribution $\widetilde{F}^{\prime}$ can be determined with the help of the functional integration

$$
\exp (-\delta W)=\int D \phi^{\prime} \exp \left(-\delta W\left[\phi^{\prime}\right]\right)
$$

where $\delta W$ is an effective potential. Using (13), (5) we obtain

$$
\delta \widetilde{F}^{\prime}=-i N_{c} N_{f} \int \frac{d^{4} k}{(2 \pi)^{4}} \ln \left[c_{0} \omega^{2}-c \vec{k}^{2}-m^{2}\right] .
$$

Again within the Matsubara technique one still should do the replacement $\omega \rightarrow \omega_{n}=2 \pi i n T,-i \int \frac{d^{4} k}{(2 \pi)^{4}} \rightarrow T \sum_{n=-\infty}^{n=\infty} \int \frac{d^{3} k}{(2 \pi)^{3}}$. We dropped an infinite constant term in (14). However expression (14) still contains a divergent contribution. To remove the regular term that does not depend on the closeness to the critical point we find the temperature derivative of (14) (entropy per unit volume):

$$
\delta \widetilde{S}^{\prime}=\frac{\partial \delta \widetilde{F}^{\prime}}{\partial T}=\frac{\partial m^{2}}{\partial T} N_{c} N_{f} \sum_{k}\left|\phi_{k}^{\prime}\right|^{2} .
$$

The contribution of fluctuations to the specific heat density is then given by

$$
\begin{aligned}
\widetilde{C}_{V}^{\prime} & =-T \frac{\partial m^{2}}{\partial T} N_{c} N_{f} \frac{\partial \sum_{k}\left|\phi_{k}^{\prime}\right|^{2}}{\partial T}-T \frac{\partial^{2} m^{2}}{\partial T^{2}} N_{c} N_{f} \sum_{k}\left|\phi_{k}^{\prime}\right|^{2} \\
& \simeq-T \frac{\partial m_{\mathrm{MF}}^{2}}{\partial T} N_{c} N_{f} \frac{\partial \sum_{k}\left|\phi_{k}^{\prime}\right|^{2}}{\partial T}, \frac{\partial m_{\mathrm{MF}}^{2}}{\partial T} \simeq-\eta_{\mathrm{MF}} a_{0} / T_{c} .
\end{aligned}
$$


In the second line (15) we remained only the terms quadratic in fluctuation fields, i.e. we assumed $\left|\frac{\partial m_{\mathrm{MF}}^{2}}{\partial T}\right| \gg\left|\frac{\partial \delta m^{2}}{\partial T}\right|$.

Knowing the contribution of fluctuations to the entropy and specific heat we may recover their contribution to the free energy and energy densities

$$
\delta \widetilde{F}^{\prime}=\left.\int_{0}^{T} \widetilde{S}^{\prime}\right|_{\rho_{q}} d T, \quad \delta \widetilde{E}^{\prime}=\left.\int_{0}^{T} \widetilde{C}_{V}^{\prime}\right|_{\rho_{q}} d T .
$$

With (15) - (17) one may recover fluctuation contribution to all thermodynamic quantities.

Now we are able to calculate the quantity $i G^{--}(X=0)=\sum_{k}\left|\phi_{k}^{\prime}\right|^{2}$ and its temperature derivative, where $i G^{--}(X)$ is the time-ordered Green function. Using the Matsubara replacement $\omega \rightarrow \omega_{n}=2 \pi i n T$ and the relation $\sum_{n}\left(y^{2}+\right.$ $\left.n^{2}\right)^{-1}=\frac{\pi}{y} \operatorname{cth}(\pi y)$, or the corresponding relation between the non-equilibrium Green functions $i G^{--}(X)$ and $\operatorname{Im} G^{r e t}$ at finite temperature, we arrive at the expression

$$
\sum_{k}\left|\phi_{k}^{\prime}\right|^{2}=\frac{1}{2 \sqrt{c_{0}}} \int \frac{d^{3} k}{(2 \pi)^{3}} \frac{1}{\sqrt{c \vec{k}^{2}+m^{2}}} \mathrm{cth}\left(\frac{\sqrt{c \vec{k}^{2}+m^{2}}}{2 T \sqrt{c_{0}}}\right) .
$$

Using that $\operatorname{cth}(y / 2)=2 n_{B}(y)+1$, where $n_{B}(y)=\left(e^{y}-1\right)^{-1}$ are Bose occupations, and dropping the regular contribution of quantum fluctuations we obtain

$$
\sum_{k}\left|\phi_{k}^{\prime}\right|_{T}^{2}=\frac{1}{\sqrt{c_{0}}} \int \frac{d^{3} k}{(2 \pi)^{3}} \frac{1}{\sqrt{c \vec{k}^{2}+m^{2}}} n_{B}\left(\frac{\sqrt{c \vec{k}^{2}+m^{2}}}{T \sqrt{c_{0}}}\right) .
$$

By index " $T$ " we indicate the thermal contribution.

The integration is performed analytically in the limiting cases. Let $m \gg$ $T \sqrt{c_{0}}$. Then $n_{B}(y) \simeq e^{-y}$. In the very same approximation one has $c \vec{k}^{2} \ll$ $m^{2}$ for typical momenta. Then

$$
\sum_{k}\left|\phi_{k}^{\prime}\right|_{T}^{2}=\frac{c_{0}^{1 / 4}}{8 m}\left(\frac{2 m T}{\pi c}\right)^{3 / 2} \exp \left(-\frac{m}{T \sqrt{c_{0}}}\right), m \gg 2 T \sqrt{c_{0}} .
$$

In the opposite limiting case, $m \ll 2 T \sqrt{c_{0}}$, there are two contributions to the integral (19), from the region of typical momenta $c \vec{k}^{2} \sim m^{2}$ and from the region $c \vec{k}^{2} \gg m^{2}$. They can be easily separated, if one calculates the auxiliary quantity $\frac{\partial \sum_{k}\left|\phi_{k}^{\prime}\right|^{2}}{\partial T}$. In the region $c \vec{k}^{2} \sim m^{2}$ one may use an approximation $n_{B}(y) \simeq 1 / y$ and

$$
\frac{\partial \sum_{k}\left|\phi_{k}^{\prime}\right|^{2}}{\partial T}\left[c \vec{k}^{2} \sim m^{2}\right] \simeq-\left(4 \pi c^{3 / 2}\right)^{-1} T \frac{\partial m}{\partial T} .
$$


The region $c \vec{k}^{2} \gg m^{2}$ yields a regular term

$$
\sum_{k}\left|\phi_{k}^{\prime}\right|^{2}\left[c \vec{k}^{2} \gg m^{2}\right] \simeq \frac{T^{2}}{12} \sqrt{\frac{c_{0}}{c^{3}}}, \quad \frac{\partial \sum_{k}\left|\phi_{k}^{\prime}\right|^{2}}{\partial T}\left[c \vec{k}^{2} \gg m^{2}\right] \simeq \frac{T}{6} \sqrt{\frac{c_{0}}{c^{3}}} .
$$

General expression for the fluctuation contribution to the specific heat is given by the first line (15) and can be resolved with the help of the self-consistent solution of (11), (19) (or (20), (21), (22) in the limiting cases). Assuming for rough estimates that fluctuations can be described perturbatively and putting $m^{2} \simeq m_{\mathrm{MF}}^{2}=\eta_{\mathrm{MF}}|a|$, from the second line of (15) we find for $T \ll \frac{m_{\mathrm{MF}}}{\sqrt{c_{0}}}$ :

$$
\begin{aligned}
\widetilde{C}_{V}^{\prime} & \simeq \frac{N_{c} N_{f} \eta_{\mathrm{MF}}^{7 / 4} c^{1 / 4} T^{3 / 2}}{4 \pi^{3 / 2} \sqrt{2} c_{0}^{1 / 4}|t|^{1 / 4} T_{c}^{2}} \\
& \times\left(\frac{a_{0}}{c}\right)^{7 / 4}\left(1+\frac{2|t| T_{c}}{T}\right) \exp \left(-\frac{\sqrt{\eta_{\mathrm{MF}} a_{0}|t|}}{T \sqrt{c_{0}}}\right)
\end{aligned}
$$

and for $T \gg \frac{m_{\mathrm{MF}}}{\sqrt{c_{0}}}$ :

$$
\widetilde{C}_{V}^{\prime} \simeq \frac{N_{c} N_{f} \eta_{\mathrm{MF}}^{3 / 2} T^{2}}{8 \pi T_{c}^{2}|t|^{1 / 2}}\left(\frac{a_{0}}{c}\right)^{3 / 2}+\frac{N_{c} N_{f} \eta_{\mathrm{MF}} T^{2}}{6 T_{c}} \frac{a_{0}}{c}\left(\frac{c_{0}}{c}\right)^{1 / 2} .
$$

In the weak coupling limit for the CFL phase $a_{0} / c=6 \pi^{2} T_{c}^{2} /(7 \zeta(3) / 8)$, $c_{0}=3 c, \eta_{\mathrm{MF}}=2 / 3$. Eq. (22) holds in the low temperature limit $T \ll$ $\pi(|t| / 3)^{1 / 2} T_{c}$, whereas eq. (24) is valid in the opposite limit. The first term in (24) dominates over the second one for $|t|<0.7$, i.e., in the whole region of validity of (24) the main term is the first one. Its singular behavior, $\sim 1 / \sqrt{|t|}$, is typical for the specific heat in the vicinity of the critical point of the second order phase transition, as in metallic superconductors.

Also in the CFL phase $\left(T<T_{c}\right)$ there is a contribution to the specific heat of Goldstone-like excitations, cf. phonons in the ordinary condensed matter. For $T \gg m_{p . G}$, where $m_{p . G}$ is the mass of the pseudo-Goldstone excitation, we get

$$
\widetilde{C}_{V}^{p . G, \prime}=\frac{N_{G} 3^{3 / 2} \pi^{2} T^{3}}{30},
$$

where $N_{G}$ is the number of pseudo-Goldstone modes. One can see that the contribution (25) is numerically small compared to those terms (cf. (22), (24)) we have evaluated for temperatures within the fluctuation region.

Now we are able to discuss the validity of the self-consistent Hartree approximation in our problem. Adding the contribution to $\Phi$ functional with two vertices produces the sun-set diagram in the di-quark self-energy. In such a 
way beyond the Hartree approximation there appears the width term in the self-energy (behaving as $-i \gamma \omega$ for small $\omega$ ). Such a term governs the slow relaxation of the order parameter in the phase transition phenomena outside the equilibrium. In our case (thermal equilibrium) this term can be dropped compared to the $c_{0} \omega^{2}$ term, which we have in the thermodynamic potential (1) from the very beginning, at least in both the low and high temperature limits. Indeed, in the low temperature limit three Green functions entering the sun-set diagram produce an extra exponentially small particle occupation factor compared to that governs the Hartree term (20). Near $T_{c}$, i.e. in the high temperature limit, we may remain only $n=0$ term in the Matsubara sum over frequencies, as we have argued, thus suppressing both the linear and the quadratic terms in $\omega$.

\section{Ginzburg - Levanyuk criterion and Ginzburg number}

Comparing the mean field (12) and the fluctuation (15) contributions to the specific heat (in the low and high temperature limiting cases one may use eqs. (22), (24)) we may estimate the fluctuation temperature $T_{\mathrm{fl},<}^{C}<T_{c}$, at which the contribution of fluctuations of the order parameter becomes to be as important as the mean field one (so called Ginzburg - Levanyuk criterion),

$$
\widetilde{C}_{V}^{\prime} \simeq \widetilde{C}_{V}^{\mathrm{MF}}, \text { for } T<T_{c}
$$

Fluctuations dominate for $T>T_{\mathrm{fl},<}^{C}$. For typical values $\mu_{q} \sim(350 \div 500) \mathrm{MeV}$ and for $T_{c} \gtrsim(50 \div 70) \mathrm{MeV}$ in the weak coupling limit from (26), (22) we estimate $T_{\mathrm{fl},<}^{C} \simeq(0.6 \div 0.8) T_{c}$. If we took into account the suppression factor $f$ of the mean field term $\propto e^{-\Delta(0) / T}$, a decrease of the mass $m$ due to the fluctuation contribution (cf. (11)), and the pseudo-Goldstone contribution (25), we would get still smaller value of $T_{\mathrm{fl},<}^{C}\left(\lesssim 0.5 T_{c}\right)$. We see that fluctuations start to contribute at temperatures when one can still use approximate expressions (22), (20) valid in the low temperature limit. Thus the time (frequency) dependence of the fluctuating fields is important in case of CSC.

In the condensed matter physics one usually performs calculations in the high temperature limit. In this limit one neglects the time (frequency) dependent terms considering quasi-static thermal fluctuations of the order parameter. Then the fluctuation contribution is determined with the help of the functional integration

$$
\begin{aligned}
\exp \left(-\delta F^{\prime} / T\right) & =\int D d^{\prime} \exp \left(-\delta F^{\prime}\left[d^{\prime}\right] / T\right) \\
\delta F^{\prime}\left[d^{\prime}\right] & =\sum_{i, \alpha, \vec{k}}\left(c \vec{k}^{2}+\eta|a|\right)\left(d_{\alpha, \vec{k}}^{i, \prime}\right)^{2}
\end{aligned}
$$


The integration yields

$$
\delta \widetilde{F}^{\prime}=T N_{f} N_{c} \int \frac{d^{3} k}{(2 \pi)^{3}} \ln \left[c \vec{k}^{2}+\eta|a|\right] .
$$

Comparison of eqs. (28) and (14) shows that the high temperature expression (28) is recovered, if one drops all the terms except $n=0$ in the corresponding Matsubara sum over $\omega_{n}=2 \pi i n T$ in eq. (14). The contribution of $n \neq 0$ terms to the $\sum_{k}\left|\phi_{k}^{\prime}\right|^{2}$ is suppressed in the limit $c_{0} 4 \pi^{2} T^{2} \gg m^{2}$. Then one immediately arrives at the specific heat given by the first term of (24). Thus in our case one may suppress the frequency dependence of fluctuations only for $|t| \ll 1$.

Simplifying, the energy width of the fluctuation region, where the fluctuation effects prevail over the mean field ones, is usually estimated following the Ginzburg criterion. The probability of the fluctuation in the volume $V_{\mathrm{fl}}$ is given by $W \sim \exp \left(-\delta \Omega\left(V_{\mathrm{fl}}\right) / T\right)$. It is $\sim 1$ for $\delta \Omega\left(V_{\mathrm{fl}}\right) \sim T$, where $\delta \Omega\left(V_{\mathrm{fl}}\right)$ (the contribution to the thermodynamic potential in the corresponding variables) is the work necessary to prepare the fluctuation within the volume $V_{\mathrm{fl}}$. The minimal size of the fluctuation region characterized by an order parameter $d \sim \sqrt{D_{\mathrm{MF}}}$ is $\xi(T) \sqrt{2 / \eta}$, cf. (5), (8). Thus, taking $\delta \Omega\left(V_{\mathrm{fl}}\right)=\delta \Omega_{\mathrm{MF}}\left(V_{\mathrm{fl}}\right)=T_{\mathrm{fl}}^{\mathrm{G}}$ for the typical temperature $T_{\mathrm{fl}}^{\mathrm{G}}$, when fluctuations start to dominate, we obtain

$$
T_{\mathrm{fl}}^{\mathrm{G}} \simeq \frac{a^{2}}{2 b} \frac{4 \pi\left(\sqrt{2 / \eta} \xi\left(T_{\mathrm{fl}}^{\mathrm{G}}\right)\right)^{3}}{3} \simeq \frac{4 \pi a_{0}^{1 / 2} c^{3 / 2}\left|t\left(T_{\mathrm{fl}}^{\mathrm{G}}\right)\right|^{1 / 2}}{\eta^{3 / 2} b} \frac{\sqrt{2}}{3} .
$$

Although the above estimate is very rough we took care of all the numerical factors. This allows us to notice that the value $T_{\mathrm{fl},<}^{C}$ estimated from (26), if one uses eq. (24) for $\widetilde{C}_{V}^{\prime}$ remaining there only the first term, is $T_{\mathrm{fl},<}^{C} \simeq$ $0.5\left|t\left(T_{\mathrm{fl},<}^{C}\right) / t\left(T_{\mathrm{fl}}^{\mathrm{G}}\right)\right|^{1 / 2} T_{\mathrm{fl}}^{\mathrm{G}}$ for $N_{c}=N_{f}=3$. All the dependencies on the parameters in expressions for $T_{\mathrm{fl},<}^{C}$ and $T_{\mathrm{fl}}^{\mathrm{G}}$ were proven to be essentially the same.

Fluctuation region is determined by the Ginzburg number $G i=\mid T_{c}-$ $T_{\mathrm{fl}}^{\mathrm{C}} \mid / T_{c}$. The larger the value $G i$ is the broader is the fluctuation region. For clean conventional superconductors [15] $G i \simeq A\left(T_{c} / \mu\right)^{4} \sim 10^{-12} \div 10^{-14}$, $A \sim 80$, whereas for superfluid $\mathrm{He}^{4}$ and in our case $G i \sim 1$ (since $A \sim 500$ and $T / \mu_{q}$ can be as large as $\frac{1}{3} \div \frac{1}{5}$ in favorable cases). The role of fluctuations increases in cases, when the effective dimensionality of the droplet decreases or/and when the quark mean free path decreases. Both possibilities result in a significant increase of the $G i$-number. In the case of quark droplets of the typical size $L \ll \xi$ one deals with the zero-dimensional system, $G i \propto \xi^{3} / L^{3}$ and the fluctuation contribution to the specific heat behaves as $\widetilde{C}_{V}^{\prime} \sim 1 / t^{2}$, for $G i \ll|t| \ll 1$. For dirty superconductors $G i$ increases $\propto 1 /\left(p_{F e} l_{e}\right)$, where $p_{F e}$ is the electron Fermi momentum and $l_{e}$ is the electron mean free path. A 
significant decrease of the quark mean free path is expected due to the presence of the hadron impurities inside the quark droplet. Thus, there is still a variety of possibilities for a further enhancement of superconducting fluctuations.

\section{Fluctuations of the gap above $T_{c}$}

All above expressions for fluctuating quantities (except the vanishing of the pseudo-Goldstone contribution above $T_{c}$ and the appearance of an extra diquark decay contribution to the width) are also valid for $T>T_{c}$, if one puts $D_{c}=0$ in general expressions or $m_{\mathrm{MF}}^{2}=a>0, D_{c}=0, \eta=\eta_{\mathrm{MF}}=1$ in the corresponding approximate expressions. Above $T_{c}$ we should compare the fluctuation contribution to a thermodynamic quantity with the quark and gluon contributions of the normal state of the quark-gluon plasma. In the weak coupling limit for the specific heat density of the quark-gluon plasma one has, cf. [22],

$$
\widetilde{C}_{V}^{q g} \simeq 6 \mu_{q}^{2} T+\frac{42 \pi^{2} T^{3}}{15}+\frac{32 \pi^{2} T^{3}}{15} .
$$

The first two terms are quark contributions and the third term is the gluon contribution. We omitted a contribution of strange quarks which is rather small for $T<m_{s}$ and we neglected the temperature dependent effective gluon mass. We also disregarded the $\alpha_{s}$ corrections to the quark and gluon terms since such corrections were not taken into account for condensate quantities. If we included the quark-gluon masses that appear in the framework of the approach [23] matching the quasiparticle quark-gluon description and the lattice results, we would get even smaller contribution of $\widetilde{C}_{V}^{q g}$, that is in favor of fluctuation effects.

Above $T_{c}$, eqs. (22) and (24) yield rather smooth functions of $T$ except the region $t \ll 1$. The fluctuation region is rather wide since $\widetilde{C}_{V}^{\prime} \sim \widetilde{C}_{V}^{q g}$ even at $T$ essentially larger then $T_{c}$. The appearance of an extra channel of the di-quark decay width, $2 \gamma_{\mathrm{dec}} \omega$, beyond the Hartree approximation does not qualitatively change the situation since $\gamma_{\mathrm{dec}}$ is a regular function of $T, \gamma_{\mathrm{dec}} \propto$ $T-T_{c}$, and vanishes in the critical point. Thus the applicability of the high temperature limit (one can put $\omega_{n}=0$ in the Matsubara sum) is preserved in a wide temperature region, $T \sim T_{c}$, [15]. Only far above $T_{c}$ situation might be changed. The main uncertainty comes from the values of coefficients (2) which were derived for $T$ rather near $T_{c}$ and in the weak coupling limit. Bearing all this in mind we estimate the value of the fluctuation temperature $T_{\mathrm{fl},>}^{C} \sim 2 T_{c}$ for typical value $T_{c} / \mu_{q} \sim 0.1 \div 0.3$. The higher this ratio is, the stronger is the contribution of fluctuations at the given ratio $T / T_{c}$. Note that a decrease of $\mu_{q}$ with increase of the temperature results in an increase of the ratio $T_{c} / \mu_{q}(T)$. On the other hand $\mu_{q}$ should be at least larger than zero temperature gap in order one could speak of any pairing fluctuations at given temperature.. 
Thus we see that fluctuations of the di-quark gap may essentially contribute to the thermodynamic quantities even well below $T_{c}$ and above $T_{c}$.

\section{Assumptions which we have done}

Note that several simplifying assumptions were done. The coefficients (2) were derived for $|t| \ll 1$ in the weak coupling limit neglecting the strange quark mass, but applied in a wider temperature region for may be not sufficiently large $\mu_{q}$. Only Gaussian fluctuations were taken into account within the self-consistent Hartree approximation. Thus, di-quark width effects were assumed to be suppressed. We used the expansion of the thermodynamic potential up to the fourth order terms in the mean field neglecting a small jump in the order parameter due to gluon fluctuations. As was argued in [18] gluon fluctuations may also contribute but in a more narrow vicinity of $T_{c}$ than the order parameter fluctuations (for the values of the ratio $T_{c} / \mu_{q} \sim 0.15 \div 0.3$, which we are interested here). We incorporated in the thermodynamic quantities only the terms which have a tendency to an irregular behavior near $T_{c}$, whereas above $T_{c}$ the short range correlations begin to be more and more important with the increase of the temperature. Therefore one certainly should be cautious applying above rough estimates outside the region of their quantitative validity. However, as we know from the experience of the condensate matter physics, see [15], such extrapolation equations work usually not too bad even for temperatures well below and above $T_{c}$.

\section{Fluctuations of temperature, density, magnetic susceptibility}

So far we have discussed the specific behavior of fluctuations of the order parameter at fixed temperature and density. There are also fluctuations of the temperature and the local quark density. They are statistically independent quantities [19], $<\delta T \delta \rho_{q}>=0$, and their mean squares are

$$
<(\delta T)^{2}>=\frac{T^{2}}{V_{\mathrm{fl}} \widetilde{C}_{V}}, \quad<\left(\delta \rho_{q}\right)^{2}>=\frac{T \rho_{q}}{V_{\mathrm{fl}}}\left(\frac{\partial \rho_{q}}{\partial P}\right)_{T} .
$$

The averaging is done over the volume, $P$ is the pressure, $V_{\mathrm{fl}}$ is as above the volume related to the fluctuation. In the limiting case $\widetilde{C}_{V}^{\prime} \ll \widetilde{C}_{V}^{q g}$, we obtain $<(\delta T)^{2}>/ T^{2} \simeq \rho_{q} /\left(N_{q}^{\mathrm{fl}} \widetilde{C}_{V}^{q g}\right)$, where $N_{q}^{\mathrm{fl}}$ is the number of quarks involved in the volume $V_{\mathrm{ff}}$. Thus, far from the critical point the contribution of fluctuations is suppressed as $\sqrt{<(\delta T)^{2}>/ T^{2}} \propto 1 / \sqrt{N_{q}^{\mathrm{f}}}$. This standard fluctuation behavior is essentially changed for $\widetilde{C}_{V}^{\prime} \gtrsim \widetilde{C}_{V}^{q g}$. Then we get even larger suppression of the temperature fluctuations. For $|t| \ll 1$ in the high temperature limit we obtain $\sqrt{<(\delta T)^{2}>/ T_{c}^{2}} \sim|t| \rightarrow 0$ for $|t| \rightarrow 0$, where we assumed that fluctuations are most probable within the typical fluctuation 
volume $V_{\mathrm{fl}}=4 \pi \xi^{3} / 3$ and $\xi \propto 1 / \sqrt{|t|}$ for $t \rightarrow 0$. Thus, if the system is at the temperature $T$ in a narrow vicinity of $T_{c}$, fluctuations of the temperature are significantly suppressed. Being formed at $0<-t \ll 1$ the condensate region evolves very slowly, since the heat transport is then delayed (the typical evolution time is roughly $\tau \propto 1 / \sqrt{|t|}$ ). At temperatures $T$ outside a narrow vicinity of $T_{c}$, fluctuations of the temperature resulting in a significant decrease of the temperature in the volume $V_{\mathrm{fl}}$ are rather probable, $\sqrt{\left\langle(\delta T)^{2}\right\rangle / T_{c}^{2}} \sim 1$. This is the consequence of a very short coherence length and, thus, not too large number of quarks contained in the volume $V_{\mathrm{fl}}, \xi \simeq 0.13 /\left(T_{c} \sqrt{|t|}\right)$ being $\simeq 0.5 \mathrm{fm}$ for $T_{c} \simeq 50 \mathrm{MeV}$ and for $|t| \sim 1$. Fluctuations of the quark density are also large for typical $|t| \sim 1$, due to the smallness of $V_{\mathrm{ff}}$. Thereby, we argue that the system may produce the di-quark condensate regions of the typical size $\xi$, thus feeling the possibility of the phase transition even if its temperature and density are in average rather far from the critical values.

Other quantities associated with second derivatives of the thermodynamic potential are also enhanced near the critical point demonstrating typical $1 / \sqrt{|t|}$ behavior, cf. [21]. However numerical coefficients depend strongly on what quantity is studied. E.g. fluctuation contributions above $T_{c}$ to the color diamagnetic susceptibilities

$$
\chi_{\alpha}=-\left(\partial^{2} \delta F / \partial \overrightarrow{\mathcal{H}}_{\alpha}^{2}\right)_{\overrightarrow{\mathcal{H}}_{\alpha}=0}, \quad \overrightarrow{\mathcal{H}}_{\alpha}=\operatorname{curl} \overrightarrow{\mathcal{A}}_{\alpha},
$$

are proven to be $\ll 1$ everywhere except very narrow vicinity of the critical point. In spite of a smallness, as we know, in metals the fluctuation diamagnetism turns out to be of the order of the Pauli paramagnetism even far from the transition. Also for $T \gg T_{c}$ contribution of fluctuations to the magnetoconductivity of 2D electron systems is experimentally distinguishable, [15].

\section{How gap fluctuations may manifest in heavy ion collisions}

Anomalous behavior of fluctuations might manifest itself in the event-byevent analysis of the heavy ion collision data. In small ( $L$ ) size systems, $L<\xi$, (zero dimension case would be $L \ll \xi$ ) the contribution of fluctuations of the order parameter to the specific heat is still increased, as we have mentioned, see [15]. The anomalous behavior of the specific heat may affect the heat transport. Also kinetic coefficients are substantially affected by fluctuations due to the shortening of the particle mean free paths, as the consequence of their rescatterings on di-quark fluctuations. If thermalization happened at an initial heavy ion collision stage, and a large size system expands rather slowly, its evolution is governed by the approximately constant value of the entropy. Due to a large contribution to the specific heat (and to the entropy) of di-quark fluctuations, an extra decrease of the temperature may occur resulting in an essential slowing of the fireball expansion process (due to smaller pressure). 
Having the di-quark quantum numbers, fluctuations of the gap may affect the di-lepton production rate from the quark-gluon plasma, as it has been noted in [13]. Another question is how one can distinguish di-quark fluctuations related to the CSC from the quark fluctuations, which may relate to the deconfinement transition? Theoretically, since $T_{c}$ is, in general, different from the deconfinement transition temperature $T_{d e c}$, in assumption that $T_{c}>T_{d e c}$ there might be two anomalous fluctuation peaks related to two distinct collision energies. E.g., if the observed peak [25] in the $K^{+} / \pi^{+}$ratio at $\sim(30 \div 40) \mathrm{GeV} / \mathrm{A}$ is, indeed, associated with the deconfinement transition [26], it would be worth to seach a possibility of another peak at a somewhat higher collision energy than the first one, related to the CSC phase transition, and vice versa, if the observed peak relates to the CSC, another peak at a somewhat lower energy could relate to the deconfinement. However it remains unclear is it possible to distinguish these peaks experimentally, if $T_{c}$ and $T_{d e c}$ are rather close to each other. One needs a careful measurement of $K^{+} / \pi^{+}$ratio in the whole collision energy interval.

\section{Pairing fluctuations in hybrid stars}

Besides the crust and the hadron shell, the hybrid star contains also a quark core. Both the nucleon shell and the quark core can be in superconducting phases, in dependence on the value of the temperature. Fluctuations affect transport coefficients, specific heat, emissivity, masses of low-lying excitations and respectively electromagnetic properties of the star, like electroconductivity and magnetic field structure, e.g., renormalizing critical values of the magnetic field $\left(H_{c 1}, H_{c}, H_{c 2}\right)$. Note that the CSC is the type I superconductor with the Ginzburg-Landau parameter $\kappa<0.6$, if $T_{c}<14 \mathrm{MeV}$ (estimation is done for $\mu_{q} \simeq 400 \mathrm{MeV}$ ), and it is the type II superconductor with $\kappa$ essentially larger than one, if $T_{c}$ can indeed be large, $T_{c} \gtrsim 50 \mathrm{MeV}$, that is the case which we are interested in here, cf. [18]. Especially the type II CSC could have interesting consequences for observations, cf. [11].

The effect of thermal pion fluctuations on the specific heat and the neutrino emissivity of neutron stars was discussed in [27, 28] together with other in-medium effects, see also reviews [29, 30]. Neutron pair breaking and formation $(\mathrm{PBF})$ neutrino process on the neutral current was studied in $[31,32]$ for the hadron matter. Also ref. [32] added the proton PBF process in the hadron matter and correlation processes, and ref. [33] included quark PBF processes in quark matter. PBF processes were studied by two different methods; with the help of Bogolubov transformation for the fermion wave function $[31,33]$ and within Schwinger-Kadanoff-Baym-Keldysh formalism for nonequilibrium normal and anomalous fermion Green functions [32, 28, 29]. 
As was observed in $[32,28]$, analogously to the Direct Urca (DU) process $n \rightarrow p e \bar{\nu}$, these processes $(n \rightarrow n \nu \bar{\nu}$ and $p \rightarrow p \nu \bar{\nu})$ have a large (one-nucleon) phase space volume, if the pairing gap is $\Delta \gtrsim 1 \mathrm{MeV}$ in some density interval (in addition to that DU and PBF emissivities have similar exponential suppression factors $\propto e^{-\Delta / T}$ ). Moreover, these processes are affected by nucleonnucleon [32] and electron-electron [34, 35, 30] correlations in such a way that the emissivity of the process $n \rightarrow n \nu \bar{\nu}$ is not significantly changed but the emissivity of the process $p \rightarrow p \nu \bar{\nu}$ increases up to ten - hundred times. This enhancement is due to the fact that the square of the bare vertex for the $p \rightarrow p \nu \bar{\nu}$ reaction contains a very small $\sim c_{v}^{2} \simeq 0.006$ factor compared to the corresponding factor $\sim 1$ for the $n \rightarrow n \nu \bar{\nu}$ reaction channel. However the proton may produce the neutron-neutron hole by the strong interaction and the electron-electron hole by the electromagnetic interaction, which then may couple to the weak current. This circumstance is still ignored in a number of works, which rediscovered this process with vacuum vertices and used it within the cooling code, e.g., see [36]. It seems that an artificial a ten - hundred times suppression of the relevant process may affect their conclusions. Numerical simulation of the neutron star cooling that incorporated PBF processes with inclusion of correlation effects, as well as other relevant in-medium effects of the nucleon-nucleon and nucleon-pion interaction, like softening of pion modes, was performed in [37]. The PBF processes in the quark matter are also affected by correlation effects but, as for the case of the reaction $n \rightarrow n \nu \bar{\nu}$, in the given case the correlation effects are expected to be not so significant.

Order parameter fluctuations allow for extra neutrino processes having no exponential suppression in a broad region of temperatures near $T_{c}$.

Contribution of pairing fluctuations to the specific heat in the hadron shell is minor for the case of the neutron pairing due to a small value of $T_{c} \lesssim 1 \mathrm{MeV}$ compared to the value of the neutron chemical potential $\left(\mu_{n} \gtrsim 50 \mathrm{MeV}\right)$. Therefore in the neutron channel fluctuations of the gap are relevant only in a very narrow vicinity of the critical point. However this effect might be not so small for protons, for which the chemical potential is of the order of several $\mathrm{MeV}$, whereas the gap is of the order of one MeV. Therefore it seems that fluctuations may smear the phase transition in a rather broad vicinity of the critical point of the proton superconductivity.

Pairing fluctuation effects in the quark matter are especially important, if the critical temperature of the CSC phase transition is rather high $\left(T_{c} \gtrsim 50 \mathrm{MeV}\right)$, as is estimated for $2 \mathrm{SC}$ and CFL cases. Temperatures $\sim 10 \div 50 \mathrm{MeV}$ may indeed arise at an initial stage of the hybrid star cooling. The fluctuation contribution to the total specific heat for $T \lesssim T_{c}$ is evaluated in a line with that we have done above. As noted in ref. [15], the Aslomasov - Larkin contribution to the electro-conductivity proves to be the dominant term in the vicinity of $T_{c}$. Analogously, one may expect a significant increase of the heat conductiv- 
ity and viscosity that would result in a delay of the heat transport at the initial stage of the hybrid star cooling. Moreover, neutrino may efficiently rescatter on fluctuations that governs their drift to the hadron shell at $T \lesssim T_{c}$. These effects may be especially important for the CFL phase, since in that case all other mechanisms of the heat transport are suppressed at $T<T_{c}$. Estimation of all these effects needs however a separate study.

\section{Concluding remarks}

We would like once more to emphasize that the coefficients of the thermodynamic potential (1) obtained in the weak coupling limit might be essentially modified, in case if we applied the results to the fireball produced in a heavy ion collision or to hybrid stars, somehow changing our conclusions. E.g., the quark chemical potential decreases with the temperature. It results in an additional increase of the contribution of fluctuations, $\mathrm{cf}$. dependences of the coefficients (2) on $\mu_{q}$. Thus we may need to analyze the strong coupling limit instead of the weak coupling limit we discussed. There exist arguments that the strange quark mass $m_{s}$ is very large and due to that the phase transition in the CFL phase does not occur up to very high baryon densities [38]. If $\mu_{q}$ becomes smaller than the strange quark mass we come from the possible $3 \mathrm{SC}$ phases to the 2SC phases. A more general discussion of modifications beyond the framework of the weak coupling limit can be found in [24]. A discussion how the coefficients (1) may in general vary within the Ginzburg - Landau approach is given in [12]. Our above arguments are admittedly speculative, and mean only to demonstrate a qualitative possibility of their application to heavy ion collisions and to an initial stage evolution of the hybrid star. A more detailed treatment of the problem definitely needs a further work.

Concluding, within the Ginzburg - Landau approach we estimated the fluctuation energy region at the CSC phase transition. The quantitative estimates are based on the values of parameters derived in the weak coupling limit for fluctuations of the CFL order parameter. Qualitative results survive also for fluctuations of other possible phases. We found that the frequency dependence of fluctuations is important for CSC in a wide temperature region. Fluctuations may contribute essentially to the specific heat even at $T$ rather far below and above $T_{c}$. We estimated $T_{\mathrm{fl},<} \lesssim 0.5 T_{c}$ and $T_{\mathrm{fl},>} \sim 2 T_{c}$. Our rough estimates show that the high temperature CSC could manifest itself through fluctuations of the di-quark gap in the course of the heavy ion collisions at SIS300, if the critical temperature of the phase transition is indeed rather high, $T_{c} \gtrsim(50 \div 70) \mathrm{MeV}$. The CSC fluctuations are also relevant for an initial stage of the hybrid star evolution, if $T_{c}$ is $\sim 50 \mathrm{MeV}$. However quantitative results depend on the values of several not sufficiently known parameters and further studies are still needed to arrive at definite conclusions. 


\section{Acknowledgments}

The author thanks D. Blaschke, T. Kunihiro and M.F.M. Lutz for the discussions. He acknowledges the hospitality and support of GSI Darmstadt. The work has been supported in part by DFG (project 436 Rus 113/558/0-2), and by RFBR grant NNIO-03-02-04008. The author also acknowledges NATO Scientific Program for the support of his participation in Advanced Research Workshop in Erevan, Sept. 2003.

\section{References}

[1] D. Bailin, and A. Love, Phys. Rep. 107, 325 (1984).

[2] M. Alford, K. Rajagopal, and F. Wilczek, Phys. Lett. B 422, 247 (1998).

[3] R. Rapp, T. Schafer, E.V. Shuryak, and M. Velkovsky, Phys. Rev. Lett. 81, 53 (1998).

[4] K. Rajagopal, and F. Wilczek, hep-ph/0011333.

[5] D. Diakonov, H. Forkel, and M. Lutz, Phys. Let. B 373, 147 (1996); G.W. Carter, and D. Diakonov, Phys. Rev. D 60, 016004 (1999); R. Rapp, E. Shuryak, and I. Zahed, Phys. Rev. D 63, 034008 (2001).

[6] D. Blaschke, and C.D. Roberts, Nucl. Phys. A 642, 197 (1998); J.C.R. Bloch, C.D. Roberts, and S.M. Schmidt, Phys. Rev. C 60, 65208 (1999).

[7] M. Alford, K. Rajagopal, and F. Wilczek, Nucl. Phys. B 357, 443 (1999); T. Schafer, and F. Wilczek, Phys. Rev. Lett. 82, 3956 (1999).

[8] M. Alford, J. Berges, and K. Rajagopal, Nucl. Phys. B 558, 219 (1999).

[9] A. Schmitt, Q. Wang, and D. H. Rischke, Phys. Rev., D 66, 114010 (2002).

[10] D. Page, M. Prakash, J.M. Lattimer and A. Steiner, Phys. Rev. Lett., 852048 (2000); D. Blaschke, H. Grigorian, and D.N. Voskresensky. Astron.Astrophys. 368, 561 (2001).

[11] D. Blaschke, D. M. Sedrakian, and K. M. Shahabasian, Astron. \& Astrophys. 350, L47 (1999); D. Blaschke, T. Klahn, and D.N. Voskresensky, Ap. J. 533, 406 (2000); D.M. Sedrakian, D. Blaschke, K.M. Shahabasyan, and D.N. Voskresensky, Astrofizika 44, 443 (2001).

[12] K. Iida, and G. Baym, Phys. Rev. D 63, 074018 (2001); Phys. Rev. D 65, 014022 (2002); Phys. Rev. D 66, 014015 (2002).

[13] M. Kitazawa, K. Koide, T. Kunihiro, and Y. Nemoto, Phys. Rev. D 65, 091504 (2002); hep-ph/0309026.

[14] D.Voskresensky, nucl-th/0306077.

[15] A. Larkin, and A. Varlamov, cond-mat/0109177.

[16] I. Giannakis, and H.-c. Ren, Phys. Rev. D 65, 054017 (2002).

[17] R. Pisarski, and D. Rischke, Phys. Rev. D 60, 094013 (1999).

[18] I. Giannakis, and H.-c. Ren, hep-ph/0305235.

[19] L.D. Landau, and E.M. Lifshiz, Statistical Physics, part I, Pergamon press 1958.

[20] Yu.B. Ivanov, J. Knoll, and D.N. Voskresensky, Nucl. Phys. A657, 413 (1999).

[21] E.M. Lifshiz, and L.P. Pitaevsky, Statistical Physics, part II, Pergamon press 1980.

[22] B. Muller, hep-th/9211010, Ann. Rev. Nucl. Part. Sci. 46, 71 (1996). 
[23] B. Kampfer, A. Peshier, and G. Soff, hep-ph/0212179.

[24] R. Pisarski, Phys. Rev. C 62, 035202 (2002).

[25] S.V. Afanasiev et al., NA49 Collab., Phys. Rev. C66, 054902 (2002); C. Alt et al., nuclex/0305017.

[26] M. Gazdzicki, and M.I. Gorenstein, Acta Phys. Polon., B30, 2705 (1999).

[27] D.N. Voskresensky, and A.V. Senatorov, JETP 63, 885 (1986).

[28] A.V. Senatorov, and D.N. Voskresensky, Phys. Lett. B184, 119 (1987).

[29] A.B. Migdal, E.E. Saperstein, M.A. Troitsky, and D.N. Voskresensky, Phys. Rep. 192, 179 (1990).

[30] D.N. Voskresensky, in "Physics of neutron star interiors", eds. D. Blaschke, N.K. Glendenning, A. Sedrakian, Springer, Heidelberg, 2001, p. 467; astro-ph/0101514.

[31] E.G. Flowers, M. Ruderman, and P.G. Sutherland, Astroph. J. 205, 541 (1976).

[32] D.N. Voskresensky, and A.V. Senatorov, Sov. J. Nucl. Phys. 45, 411 (1987).

[33] P. Jaikumar, and M. Prakash, Phys. Lett. B516, 345 (2001).

[34] D.N. Voskresensky, E.E. Kolomeitsev, B. Kampfer, JETP 87, 211 (1998).

[35] L.B. Leinson, Phys. Lett. B473, 318 (2000).

[36] D.G. Yakovlev, A.D. Kaminker, O.Y. Gnedin, and P. Haensel, Phys. Rep. 354, 1 (2001); D.G. Yakovlev, O.Y. Gnedin, A.D. Kaminker, K.P. Levenfish, and A.Y. Potekhin, astro$\mathrm{ph} / 0306143$.

[37] C. Schaab, D. Voskresensky, A.D. Sedrakian, F. Weber, and M.K. Weigel, Astron. Astrophys., 321, 591 (1997), astro-ph/9605188.

[38] C. Gocke, D. Blaschke, A. Khalatyan, and H. Grigorian, hep-ph/0104183. 